\title{
Usefulness of postoperative high-sensitive troponin T measurement and implications for defining type 5 infarction
}

\author{
Lars Niclauss $^{1}$, Raymond Pfister ${ }^{1}$, Dominique Delay ${ }^{2}$, Piergiorgio Tozzi ${ }^{3}$, Matthias \\ $\mathrm{Kirsch}^{3}$, and rené prêtre ${ }^{1}$ \\ ${ }^{1} \mathrm{CHUV}$ \\ ${ }^{2}$ Hopital du Valais \\ ${ }^{3}$ Centre Hospitalier Universitaire Vaudois
}

June 4, 2021

\begin{abstract}
Background and aim of the study Guidelines on myocardial revascularization indicate for type V myocardial infarction (MI) that postoperative troponin elevations need not be exclusively ischemic but may also be caused by direct epicardial injury. Additional complexity arises from the introduction of high-sensitive troponin markers. The present study attempts to contribute to the understanding of postoperative high-sensitive cardiac troponin $\mathrm{T}$ (hs-cTnT) increase. Methods Type of surgery, potential factors affecting the postoperative hs-cTnT increase, and possible thresholds indicative of type V MI were analyzed. Results Among 400 included patients, $2.8 \%$ had intervention-related ischemia analogous to the type V MI definition. Receiver-operating characteristics confirmed good discriminatory power for hs-cTnT and creatine kinase myocardial band (CK-MB), with ischemia indicating thresholds for hs-cTnT (1705.5 ng/l) and for CK-MB (113 U/l). The median postoperative hs-cTnT/CK-MB increase differed significantly depending on the type of surgery, with the highest increase after mitral valve and the lowest after off-pump coronary surgery. Regression analysis confirmed Maze procedure $(\mathrm{p}<0.001)$, cardiopulmonary bypass time $(\mathrm{p}=0.03)$, emergency indications $(\mathrm{p}=0.01)$ and blood transfusion $(\mathrm{p}=0.02)$ as significant factors associated with hs-cTnT increase. In contrast, CKMB increase was also associated with mortality $(\mathrm{p}=0.002)$. Intra-pericardial defibrillation was the only ischemia-independent factor additionally associated with proposed thresholds $(\mathrm{p}<0.001)$. Conclusions The present results confirm the influence of the type of surgery and other intervention-related parameters on the postoperative hs-cTnT increase. Type V MI-indicating thresholds may require reassessment, especially using high-sensitive markers.
\end{abstract}

Title : Usefulness of postoperative high-sensitive troponin $\mathrm{T}$ measurement and implications for defining type 5 infarction

Lars Niclauss 1

Raymond Pfister 1

Dominique Delay 2

Piergiorgio Tozzi 1

Matthias Kirsch 1

René Prêtre 1

1 Cardiovascular Surgery, University Hospital of Lausanne (CHUV), Rue du Bugnon 46, CH-1011 Lausanne 2 Cardiac surgery, Cantonal Hospital Sion, Av. du Grand-Champsec 80, CH-1951 Sion

Corresponding author: 
Lars Niclauss / Cardiac surgery -CHUV / Rue du Bugnon 46 / CH-1011 Lausanne

Tel: +41 795561690 / Fax: +41213142278

lars.niclauss@chuv.ch

Funding statement

No funding was received for this work.

Conflict of interest none declared

Word count: 3262

\section{Abstract \\ Background and aim of the study}

Guidelines on myocardial revascularization indicate for type V myocardial infarction (MI) that postoperative troponin elevations need not be exclusively ischemic but may also be caused by direct epicardial injury. Additional complexity arises from the introduction of high-sensitive troponin markers. The present study attempts to contribute to the understanding of postoperative high-sensitive cardiac troponin $\mathrm{T}$ (hs-cTnT) increase.

\section{Methods}

Type of surgery, potential factors affecting the postoperative hs-cTnT increase, and possible thresholds indicative of type V MI were analyzed.

Results

Among 400 included patients, 2.8\% had intervention-related ischemia analogous to the type V MI definition. Receiver-operating characteristics confirmed good discriminatory power for hs-cTnT and creatine kinase myocardial band (CK-MB), with ischemia indicating thresholds for hs-cTnT (1705.5 ng/l) and for CKMB (113 U/l). The median postoperative hs-cTnT/CK-MB increase differed significantly depending on the type of surgery, with the highest increase after mitral valve and the lowest after off-pump coronary surgery. Regression analysis confirmed Maze procedure $(\mathrm{p}<0.001)$, cardiopulmonary bypass time $(\mathrm{p}=0.03)$, emergency indications $(\mathrm{p}=0.01)$ and blood transfusion $(\mathrm{p}=0.02)$ as significant factors associated with hs-cTnT increase. In contrast, CK-MB increase was also associated with mortality $(\mathrm{p}=0.002)$. Intra-pericardial defibrillation was the only ischemia-independent factor additionally associated with proposed thresholds $(\mathrm{p}<0.001)$.

\section{Conclusions}

The present results confirm the influence of the type of surgery and other intervention-related parameters on the postoperative hs-cTnT increase. Type V MI-indicating thresholds may require reassessment, especially using high-sensitive markers.

\section{Introduction}

The European society of cardiology (ESC) state that "for the detection of type 5 myocardial infarction (MI), a specific cut-off value for all procedures and all cardiac troponin (cTn) assays is difficult to define" [1]. According to them, coronary artery bypass grafting (CABG) - related MI is defined by an increase of cTn more than 10 times the $99^{\text {th }}$ percentile upper reference limit (URL) during the first 48 hours after surgery (in patients with normal baseline cTn) with at least one of the following 1). Development of new $\mathrm{Q}$ waves, 2). Angiographic documented graft or coronary occlusion, 3). Imaging evidence of loss of viable myocardium or regional wall motion abnormality [1]. The authors point out the existing complexity of type 5 MI diagnosis: "Postoperatively observed ST-segment deviations and T-wave changes as well as isolated cTn elevations indicate direct procedural epicardial injury rather than myocardial ischemia" [1]. 
The introduction of high-sensitive cTn assays has led to additional ambiguity. Several assays exist for the detection of two different regulatory proteins of the tropomyosin complex based on high-affinity antibodies specific for troponin T or I. An increase in sensitivity of the corresponding tests is usually accompanied by a loss of specificity, despite improved analytical performance. Accordingly, it appears difficult to classify postoperative high-sensitive cTn values with regard to the clinical course.

The aim of this study, intentionally including different types of cardiac surgery, is to investigate associations between postoperative high-sensitive cardiac troponin $\mathrm{T}$ (hs-cTnT) rise and clinical outcome in terms of adverse events and other possible (surgery-related) factors. In addition, an attempt is made to define thresholds indicative of surgically induced myocardial ischemia, thus contributing to the general understanding of postoperatively elevated cardiac enzymes.

\section{Materials and Methods}

The institutional review board ensured ethics approval in accordance with international recommendations. According to the decision of the Ethics Committee, (protocol number 2016-01884) patient consent was waived.

Patients were operated by two surgeons in 2016 and 2017. All major cardiac interventions, except surgery for terminal heart failure (assist devices, transplantation) or acute aortic disease (dissection), were included. Follow-up focused on 30 days after surgery and documented adverse events: Mortality, type 5 MI (which requires a significant hs-cTnT increase by analogy with ESC guidelines, although the threshold needs to be further defined), stroke, changes in ventricular function, and new ECG modifications.

Other parameters that could have an impact on enzyme elevation were also considered: Demographic and risk factors, i.e., preoperative MI, European System for Surgical Cardiac Risk Evaluation (EuroSCORE II), renal function, etc., direct surgery-related factors, i.e., type of cardioplegia, cross-clamp and cardiopulmonary bypass time, blood transfusions, intra-pericardial defibrillation, etc., and type of surgery.

Cardiac enzymes, i.e. hs-cTnT and creatine kinase myocardial band (CK-MB), were recorded before and 1 , 6, 12, 24 to at least 48 hours after surgery. The hs-cTnT assay used (Elecsys Troponin T high sensitive by Roche, Basel, Switzerland) has the following specifications: $99^{\text {th }}$ percentile URL is 14 nanogram per liter (ng/l) with a precision value (coefficient of variation [?] 10\%) of $13 \mathrm{ng} / \mathrm{l}$, a limit of detection at $5 \mathrm{ng} / \mathrm{l}$ and a blank at $3 \mathrm{ng} / \mathrm{l}[2]$. CK-MB was measured in international units and its concentration expressed in units per liter $(\mathrm{U} / \mathrm{L})$.

Pre- and postoperative echocardiography was performed in all patients, with a focus on new ventricular dysfunction (considered significant if it had worsened by at least one grade according to the EuroSCORE classification) or new regional wall motion abnormalities attributable to coronary perfusion territories [3].

Type VMI

All patients with new ECG changes (Q wave, left bundle branch block not directly surgery related) and/or with above-mentioned echocardiographic criteria with concomitant elevated postoperative hs-cTnT and CKMB were identified.

\section{Thresholds for type $V M I$}

Receiver operating characteristics (ROC), respectively the area under the ROC curves (AUROC) were determined to investigate the discriminatory power of postoperative enzyme increase to identify ischemic events. The coordinates of the ROC curves were used to define potential thresholds that were as specific as possible (while maintaining high sensitivity) for ischemic events (closest point to the upper left corner).

Determination of other non-type V MI-related factors influencing cardiac enzyme increase.

Median postoperative hs-cTnT, respectively CK-MB release was compared, depending on the type of surgery, to detect possible significantdifferences . To identify possible correlations between cardiac enzyme increase and independent parameters, linear regression analyses were performed, which also allowed comparison 
between hs-cTnT and CK-MB. Binary-coded thresholds for the hs-cTnT, respectively for the combination of hs-cTnT and CK-MB, were correlated with binary-coded independent factors in logistic regression analyses.

Statistical analysis

The SPSS 27.0 software package for Windows (SPSS Inc. Chicago, Illinois, USA) was used. Categorical variables are presented as numbers and proportions (\%). Non-normally distributed variables (KolmogorovSmirnov / Shapiro-Wilk test) are described by their median and interquartile range (IQR).

To identify significant differences in hs-cTnT increase, according to the type of surgery, the nonparametric Kruskal-Wallis test was used (a p value of less than 0.05 was considered significant). If group differences were significant, their effect size was determined by calculating the correlation coefficient, the strength of which was determined following the definition of Cohen et al [4]. Linear regression analysis was used to identify correlations between enzyme release and potential cofactors. Logistic regression analysis was used to analyze the correlation between potential hs-cTnT/CK-MB thresholds and binary coded parameters.

The authors had full access to the data and take responsibility for its integrity.

\section{Results}

400 consecutive operated patients were prospectively enrolled during the 2-year study period. Demographic data, comorbidities and risk profiles are presented in Table 1/2. All patients had 30 days follow-up. Mortality rate was $1.8 \%(\mathrm{n}=7$; three deaths were cardiac-related), stroke rate was $3.3 \%(\mathrm{n}=13)$.

\section{Type of surgery and technical aspects (Table 1/2)}

168 patients $(42 \%)$ underwent CABG, performed on-pump in $30 \%(\mathrm{n}=120)$ and off-pump in $12 \%(\mathrm{n}=48)$. 101 patients (25\%) had an isolated aortic valve replacement (AVR), 50 patients (13\%) had combined surgery $(\mathrm{AVR}+\mathrm{CABG})$, and 56 patients $(14 \%)$ had some form of mitral valve surgery (MVR). The remaining 25 patients $(6 \%$; Others) had surgery on the ascending aorta. Cold blood cardioplegia was predominantly used for myocardial protection $(68 \% ; n=271)$. Surgical parameters included blood transfusions in $4.5 \%(\mathrm{n}=18)$, intra-pericardial defibrillations in $8 \%(\mathrm{n}=31)$, septal myectomies in $18 \%(\mathrm{n}=70$, all in combination with AVR / AVR + CABG), Maze procedures in $1 \%(\mathrm{n}=4)$, and left atrial appendage closure (LAAC) in 7\% (n=28) of all patients.

\section{Type V MI}

18 patients met the criteria for perioperative MI. Of these, seven already had significant enzyme elevation preoperatively, indicating ongoing infarction. This left 11 patients to whom definite perioperative ischemia could be assigned, corresponding to a perioperative MI rate of $2.8 \%$ (3.2\% of those without preoperatively elevated hs-cTnT).

$H s-c T n T$ / CK-MB increase depending on type of surgery

The median hs-cTnT release was $769 \mathrm{ng} / \mathrm{l}$ (IQR: 483 - $1306 \mathrm{ng} / \mathrm{l}$ ). The median CK-MB release was $50 \mathrm{U} / \mathrm{l}$ (IQR: $37-71 \mathrm{U} / \mathrm{l}$ ). The Kruskal-Wallis test showed significant differences in hs-cTnT release depending on type of surgery, with the AVR-CABG and MVR groups having the highest median values. The strongest significance was found in the pairwise comparison of off-pump CABG with AVR-CABG or with MVR (Table 3) . Comparatively, the median CK-MB increase was also dependent on the type of surgery, with the MVR group consistently having the highest median rank (Table 4) .

$H s-c T n T / C K-M B$ thresholds (Arrows in Figure $A+B$ )

The AUROC confirmed good discriminatory power of more than $95 \%$ for both hs-cTnT $(\mathrm{p}<0.001,95 \% \mathrm{CI}$ : 92-99\%) and CK-MB ( $<<0.001,95 \%$ CI: 93-99\%) to identify patients with type V MI. The threshold was $1705.5 \mathrm{ng} / \mathrm{l}$ for hs-cTnT with $100 \%$ sensitivity and (1 - 0.12) $88 \%$ specificity, and $113 \mathrm{U} / \mathrm{l}$ for CK-MB with $91 \%$ sensitivity and (1 - 0.07) $93 \%$ specificity.

Associated factors 
- Linear regression revealed a significant, positivecorrelation of increased hs-cTnT with type V MI, Maze procedure, cardiopulmonary bypass time, emergency intervention, perioperative blood transfusion, and intra-pericardial defibrillation. In contrast, postoperative creatinine clearance and increasing age were inversely correlated (Table 1/2 A + Supplemental Table).

- Statistical correlations for CK-MB were somewhat similar, although creatinine clearance, emergency intervention, and blood transfusion had no effect. Moreover, the strongest correlation was for cardiopulmonary bypass time, and notably, increased CK-MB release was associated with mortality (Table 1/2A + Supplemental Table).

- Intra-pericardial defibrillation, emergency intervention, Maze procedure, mortality, and postoperative renal insufficiency were positively with hs-cTnT threshold correlated independent factors, whereas the combination of hs-cTnT and CK-MB threshold was correlated with intra-pericardial defibrillation, renal insufficiency, and blood transfusions (Table 1B) .

- After screening patients with preoperatively elevated hs-cTnT, intra-pericardial defibrillation remained as an independent parameter associated with the thresholds (Table 2B) .

\section{Conclusions}

The present study is an attempt to provide an analytical overview of postoperative cardiac enzyme release considering various influencing factors in cardiac surgery. We deliberately did not select patients but considered different operations including additional procedure-related factors to cover a representative spectrum of everyday cardiac surgery. One problem of postoperative troponin release is its multifactorial origin. Therefore, systematic classification remains a challenge. It is a difference whether the operation and the resulting trauma is limited to epicardial regions (as in CABG) or whether opening of the heart cavities is necessary (as in MVR). Comparison of median postoperative hs-cTnT (and of CK-MB) shows an increase depending on the type and complexity of surgery. This was described for non-high-sensitive troponin by Landoni et al, who showed that "different operations were associated with a different release of ... biomarkers", with the highest troponin release after MVR, analogous to results presented here [5].

Mortality was rather low (1.8\%), and little association with hs-cTnT was found, in contrast to other studies that inferred increased mortality from postoperative dose-dependent troponin release [6, 7]. However, an association between mortality and increased CK-MB release was also demonstrated here.

In what is, to our knowledge, the only analysis of postoperative (after CABG) hs-cTnT to verify type 5 MI criteria, the authors found that isolated hs-cTnT raise was not related to mortality [8]. In contrast, if ECG and/or echocardiographic criteria were present, hs-cTnT elevation above 10 times the URL ( $>140$ $\mathrm{ng} / \mathrm{l}$ ) was associated with increased 30-day and 1.8-year follow-up mortality, thus apparently confirming guidelines. Strikingly, the authors state "the level of post-operative hs-cTnT $>140 \mathrm{ng} / \mathrm{l}$ was found in $90 \%$ of all patients..." which is consistent with the present results (hs-cTnT was above $140 \mathrm{ng} / \mathrm{l}$ in $91.6 \%$ of CABG patients) [8]. This leads to the question of the usefulness of hs-cTnT determination when a possible threshold for intervention-related ischemia is exceeded in more than $90 \%$ and thus the diagnosis is essentially based on ECG and/or echocardiographic findings. Thus, a fundamental problem regarding a precise threshold definition of the type 5 MI-indicating troponin increase emerges, which should be redefined specifically for "high-sensitive" assays (in which only the detection method has become more sensitive, whereas the measured troponin $\mathrm{T}$ type remains unchanged).

To complicate matters, other factors influence troponin increase. Studies in nonsurgical patients have shown that the utility of the marker for the diagnosis and management of acute coronary syndrome in the presence of renal insufficiency is limited $[9,10]$. However, in the surgical patients studied here, a possible initial association in this regard could be excluded after sorting out those with preoperatively elevated hs-cTnT. Thus, the Maze procedure, emergency intervention, blood transfusion, prolonged cardiopulmonary bypass time, and, in particular, intra-pericardial defibrillation remain as independent parameters influencing hscTnT release. Regarding the Maze procedure, enzyme release seems to be directly measurable and dependent on the invasiveness of ablation therapy [11]. 
An analysis of troponin T measurements in 847 CABG patients concluded that although only $2 \%$ had new Q waves or left bundle branch block after surgery (comparable to the incidence recorded here),"... troponin $T$ concentrations ... (are) ... almost universally elevated and are determined by numerous factors ..."[12]. The latter included prolonged cardiopulmonary bypass time and intraoperative defibrillation, both of which were associated with increased, whereas higher postoperative glomerular filtration rate and offpump procedures were associated with decreased troponin [12]. Januzzi noted, in one of the first reviews of troponin testing after cardiac surgery, that:"... biomarker (s)... could lead to an inordinate percentage of patients diagnosed with 'acute $M I$ ' ... (with)... a sensitivity of $100 \%$... observed for post-CABG MI ... associated with a specificity of $4.2 \%$ and an alarmingly high misclassification rate..." [13].

The use of high-sensitive troponin assays has done little to change this problem. An even higher overall postoperative rise in cardiac enzyme markers may further limit its specificity. Thus, it seems important to include not only the hs-cTnT but also the CK-MB rise and, of course in accordance with guidelines, any ECG changes and/or regional wall motion abnormalities that may occur. Pointing this out and supporting these findings with further studies is of current importance, especially in view of possible reinterpretation attempts, as occurred in a recent large therapy optimization study, in which, deviating from the existing guidelines, the isolated postoperative troponin rise with a new low-threshold definition served as the sole assessment criterion of type V MI [14].

With regard to a generally applicable hs-cTnT threshold definition, a binding statement based on the results presented in this study seems difficult. The calculated high hs-cTnT cutoff of 1075.5ng/l (>120URL) results from the patients evaluated here, including those who had clearly detectable surgery-induced myocardial ischemia as defined above. With an AUROC of more than 95\%, the model has good discriminatory predictive value in the classification chosen herein for type V MI. However, only 11 patients with clearly detectable perioperative infraction events constitute the type V MI group thus defined, which certainly allows only limited generalizability.

On the other hand, the calculated concomitant CK-MB increase with its type V MI-indicating threshold seems to allow some comparability with the results of previous analyses $[15,16]$. In addition, comparison of the two markers shows that CK-MB is slightly inferior to hs-cTnT in terms of sensitivity but has higher specificity, and according to regression analysis, a stronger focus on exclusively myocardial ischemic events seems possible with simultaneous consideration of CK-MB.

In conclusion, the postoperative hs-cTnT increase is multifactorial, with particular influence of intrapericardial defibrillation, type and duration of surgery, and emergency intervention. A reevaluation of the current thresholds regarding type V MI, especially in the context of the use of high-sensitive troponin markers, should be considered, but larger prospective (multicenter) studies are needed for assessment as well as a possible redefinition.

\section{Author contributions}

L. Niclauss - Concept, Data analysis, Statistics, Drafting

R. Pfister - Data collection, Statistics

D. Delay - Data collection

P. Tozzi - Critical revision

P. Kirsch - Critical revision

R. Pretre - Approval

\section{References}

Thygesen K, Alpert JS, Jaffe AS, Chaitman BR, Bax JJ, Morrow DA et al. Task Force for the Universal Definition of Myocardial Infarction. Fourth Universal Definition of Myocardial Infarction (2018). J Am Coll Cardiol. 2018;72:2231-2264. 
Saenger AK, Beyrau R, Braun S, Cooray R, Dolci A, Freidank H et al. Multicenter analytical

evaluation of a high-sensitivity troponin T assay. Clin Chim Acta. 2011;412:748-754.

1. Nashef SA, Roques F, Michel P, Gauducheau E, Lemeshow S, Salamon R. European system For cardiac operative risk evaluation (EuroSCORE). Eur J Cardiothorac Surg. 1999;16:9-13.

2. Cohen J. "A power primer". Psychological Bulletin.1992;112:155-159.

3. G Landoni, F Pappalardo, M G Calabro, F Boroli, O Sottocorna, G Aletti et al. Myocardial necrosis biomarkers after different cardiac surgical operations. Minerva Anestesiol. 2007;73:49-56.

4. Mokhtar AT, Begum J, Buth KJ, Legare JF. Cardiac troponin T is an important predictor of mortality after cardiac surgery. J Crit Care. 2017;38:41-46.

5. Nam K, Shin KW, Kim TK, Kim KH, Kim KB, Jeon Y et al. Prognostic value of high-sensitivity troponin I after cardiac surgery according to preoperative renal function. Medicine (Baltimore) 2020;99:e20040.

6. Wang TK, Stewart RA, Ramanathan T, Kang N, Gamble G, White HD. Diagnosis of MI after CABG with high-sensitivity troponin $\mathrm{T}$ and new ECG or echocardiogram changes: relationship with mortality and validation of the universal definition of MI. Eur Heart J Acute Cardiovasc Care. 2013;2:323-333.

7. Yang G, Yao Y, Du Y, Huang J. Cardiac troponin had limited diagnostic value for acute myocardial infarction in renal insufficiency: a meta-analysis. Biomark Med. 2020;14:481-493.

8. Hsu CK, Wu IW, Chen YT, Peng CH, Tseng YJ, Chen YC et al. Value of the high-sensitivity troponin $\mathrm{T}$ assay for diagnosis of acute myocardial infarction in patients with and without renal insufficiency.2020;42:1142-1151.

9. Martinez-Comendador J, Castano M, Mosquera I, Plana JG, Gualis J, Martin CE et al. Cryoablation of atrial fibrillation in cardiac surgery: outcomes and myocardial injury biomarkers. J Cardiothorac Vasc Anesth. 2011;25:1030-1035.

10. Mohammed AA, Agnihotri AK, van Kimmenade RR, Martinez-Rumayor A, Green SM, Quiroz R et al. Prospective, comprehensive assessment of cardiac troponin $\mathrm{T}$ testing after coronary artery bypass graft surgery. Circulation. 2009;120:843-850.

11. Januzzi JL Jr. Troponin testing after cardiac surgery. HSR Proc Intensive Care Cardiovasc Anesth. $2009 ; 1: 22-32$

12. Stone GW, Kappetein AP, Sabik JF, Pocock SJ, Morice MC, Puskas J et al. Five-Year Outcomes after PCI or CABG for Left Main Coronary Disease. N Engl J Med. 2019;381:1820-1830.

13. Ramsay J, Shernan S, Fitch J, Finnegan P, Todaro T, Filloon T et al. Increased creatine kinase

MB level predicts postoperative mortality after cardiac surgery independent of new Q waves. J Thorac Cardiovasc Surg. 2005;129:300-306.

Gavard JA, Chaitman BR, Sakai S, Stocke K, Danchin N, Erhardt L et al. GUARd During Ischemia Against Necrosis (GUARDIAN) Investigators. Prognostic significance of elevated creatine Kinase MB after coronary bypass surgery and after an acute coronary syndrome: results from the GUARDIAN trial. J Thorac Cardiovasc Surg. 2003;126:807-813.

\section{Figure legend}

A: Receiver-operating characteristics of hs-cTnT increase indicating ischemic events.

B: Receiver-operating characteristics of CK-MB increase indicating ischemic events

\section{Hosted file}

Table 1.docx available at https://authorea.com/users/417908/articles/524824-usefulness-ofpostoperative-high-sensitive-troponin-t-measurement-and-implications-for-defining-type5-infarction

\section{Hosted file}


Table 3.docx available at https://authorea.com/users/417908/articles/524824-usefulness-ofpostoperative-high-sensitive-troponin-t-measurement-and-implications-for-defining-type5-infarction
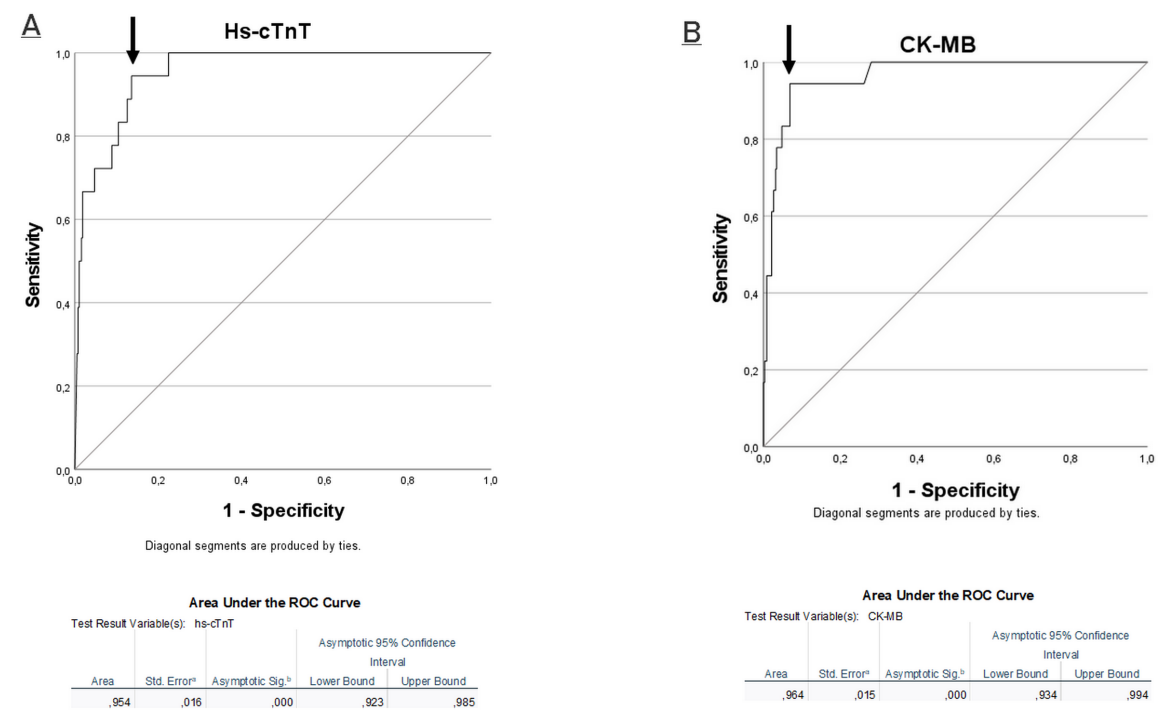

\section{Hosted file}

supplemental Table .docx available at https://authorea.com/users/417908/articles/524824usefulness-of-postoperative-high-sensitive-troponin-t-measurement-and-implications-fordefining-type-5-infarction 